\title{
A PROPERTY OF EXTREME POINTS OF COMPACT CONVEX SETS
}

\author{
MEYER JERISON
}

1. Introduction. The knowledge that a given convex set has extreme points can be very useful, and this accounts for the fame of the Krein-Milman theorem. Sometimes, more detailed information about the location of the extreme points of the set is needed. A method for obtaining such information, first used by Milman in [3], is applied here to locate the extreme points of the limit of a sequence of convex sets.

We shall be dealing with a real, locally convex, linear Hausdorff space, $E$. If $S$ is a subset of $E$, the closed convex hull of $S$ will be denoted by $S^{\mathbf{\Lambda}}$. For any $f$ in $E^{*}$ (the set of continuous linear functionals on $E$ ), we let $f_{S}=\sup _{x \in S} f(x)$. The properties of convex sets that are relevant to our problem may be summarized in one concise statement (supplied by the referee) as follows:

THEOREM 1. If $C$ is a compact convex subset of $E$, and $S \subset C$, then the following assertions are equivalent:

(i) $f_{S}=f_{C}$ for each $f \in E^{*}$;

(ii) $C=S^{\mathbf{\Lambda}}$;

(iii) the closure of $S$ includes all extreme points of $C$.

All of this is proved in [1, especially p. 84]. For instance, the assertion (iii) implies (ii) is the Krein-Milman theorem.

The technique that Milman uses in [3] is to prove (i) in order to infer (iii). Our application of it yields:

THEOREM 2. Let $\left\{K_{n}\right\}$ be a sequence of compact convex sets whose union is contained in a compact convex subset of $E$; let $A_{n}$ be the set of extreme points of $K_{n}$ for each $n$; and let $K=\lim \sup K_{n}$ and $A$ $=\lim \sup A_{n}$. Then $K \subset A \mathbf{\Delta}$. If, in addition, $K$ is convex, then $K=A \mathbf{\Delta}$, that is, $A$ contains all of the extreme points of $K$.

The limit superior is used here in the topological sense, i.e., $x \in$ $\lim \sup A_{n}$ if, and only if, every neighborhood of $x$ meets sets of the sequence $\left\{A_{n}\right\}$ with arbitrarily large index $n$. Observe that it is always a closed set.

To the best of my knowledge, Theorem 2 is new even if $E$ is finitedimensional. It has been applied in [2] to help locate the extreme

Presented to the Society, September 5, 1953; received by the editors November 23, 1953 and, in revised form, February 15, 1954. 
points of the set of generalized limits of bounded sequences.

2. Proof of Theorem 2. We treat first the case of a decreasing sequence $\left\{K_{n}\right\}$. Under that hypothesis, $K=\bigcap_{n=1}^{\infty} K_{n}$, and since it is convex, we should obtain the stronger conclusion $K=A^{\Delta}$.

Let $F_{n}$ be the closure of $\bigcup_{m=n}^{\infty} A_{m}$, so that $A=\bigcap_{n=1}^{\infty} F_{n}$. Since $F_{n} \subset K_{n}$ for each $n, A \subset K$, and therefore, for each $f \in E^{*}, f_{A} \leqq f_{K}$. The desired conclusion will follow from Theorem 1 if we succeed in reversing this inequality. By definition, $F_{n}$ contains all of the extreme points of $K_{n}$, and this implies that $f_{F_{n}}=f_{K_{n}}$. Since $F_{n}$ is compact, it contains a point $x_{n}$ such that $f\left(x_{n}\right)=f_{F_{n}}$. The sequence $\left\{x_{n}\right\}$ has a cluster point $x_{0}$ which is necessarily in $A$, and

$$
f_{A} \geqq f\left(x_{0}\right)=\lim _{n \rightarrow \infty} f\left(x_{n}\right)=\lim _{n \rightarrow \infty} f_{K_{n}} \geqq f_{K} .
$$

(The limit exists because $f_{K_{n}}$ is a monotone sequence bounded by $f_{K}$.)

The general case is handled by applying the result already obtained to the decreasing sequence $\left\{F_{n}^{\mathbf{\Lambda}}\right\}$ of compact convex sets. Since all of the extreme points of $F_{n}^{\mathbf{A}}$ are in the closed set $F_{n}$ (Theorem 1 (iii)), we have $\lim \sup \left(F_{n}^{\mathbf{\Lambda}}\right)=\left(\lim \sup F_{n}\right)^{\mathbf{\Lambda}}=\left(\bigcap_{n=1}^{\infty} F_{n}\right)^{\mathbf{\Lambda}}=A^{\mathbf{\Lambda}}$. But $K_{n}$ $=A_{n}^{\mathbf{\Lambda}} \subset F_{n}^{\mathbf{\Lambda}}$, and therefore,

$$
K=\lim \sup K_{n} \subset \lim \sup F_{n}^{\mathbf{\Delta}}=A^{\mathbf{\Delta}} .
$$

Since $A \subset K, A^{\Delta} \subset K$ if $K$ is convex, which completes the proof of the theorem.

The theorem has been so formulated as to include the case of an increasing sequence $\left\{K_{n}\right\}$, where we conclude that $A^{\mathbf{\Delta}}$ is the closure of $\bigcup_{n=1}^{\infty} K_{n}$. Also, in the general case, $\bigcap_{n=1}^{\infty} K_{n} \subset A^{\mathbf{\Lambda}}$. It may be observed, in conclusion, that the sequence of sets can be replaced by a directed system of sets.

\section{REFERENCES}

1. N. Bourbaki, Espaces vectoriels topologiques, Actualités Scientifiques et Industrielles, no. 1189, Paris, 1953.

2. M. Jerison, The set of all generalized limits of bounded sequences, to be published.

3. D. Mil'man, Isometry and extremal points, Doklady Akad. Nauk SSSR (N.S.) vol. 59 (1948) pp. 1241-1244.

PuRdue University 